\title{
Stream depletion rate with horizontal or slanted wells in confined aquifers near a stream
}

\author{
P.-R. Tsou ${ }^{1}$, Z.-Y. Feng ${ }^{1}$, H.-D. Yeh ${ }^{2}$, and C.-S. Huang ${ }^{2}$ \\ ${ }^{1}$ Department of Soil and Water Conservation, National Chung Hsing University, Taichung 402, Taiwan \\ ${ }^{2}$ Institute of Environmental Engineering, National Chiao Tung University, Hsinchu 30010, Taiwan
}

Received: 22 March 2010 - Published in Hydrol. Earth Syst. Sci. Discuss.: 15 April 2010

Revised: 4 July 2010 - Accepted: 27 July 2010 - Published: 6 August 2010

\begin{abstract}
Pumping in a vertical well may produce a large drawdown cone near the well. In this paper, the solution is first developed for describing the groundwater flow associated with a point source in a confined aquifer near a stream. Based on the principle of superposition, analytical solutions for horizontal and slanted wells are then developed by integrating the point source solution along the well axis. The solutions can be simplified to quasi-steady solutions by neglecting the exponential terms to describe the late-time drawdown, which can provide useful information in designing horizontal well location and length. The direction of the well axis can be determined from the best SDR subject to the drawdown constraint. It is found that hydraulic conductivity in the direction perpendicular to the stream plays a crucial role in influencing the time required for reaching quasisteady SDR. In addition, the effects of the well length as well as the distance between the well and stream on the SDR are also examined.
\end{abstract}

\section{Introduction}

Pumping groundwater near a stream can produce a large amount of water which may partially or totally flow from the stream after a certain period of pumping time. The use of pumping well near a stream is therefore a measure in solving the water deficiency problem, especially in an urgent need. A good example is the event happened to the Taoyuan county of Taiwan after the typhoon Matsa occurring on 5 August 2005. The Shihmen reservoir, located at northern part of Taiwan, is the major source of water supply to the Taoyuan county. After the typhoon, the turbidity of the water in Shihmen reservoir reaches $25000 \mathrm{NTU}$ which exceeds the allowable tur-

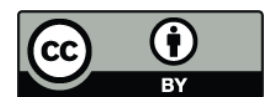

Correspondence to: Z.-Y. Feng

(tonyfeng@nchu.edu.tw) bidity of 50 NTU that the water treatment plant can manage. This resulted in more than 630000 households without having water supply. At that time, the water company drilled wells near the Taoyuan canal for acquiring the low turbid groundwater which in fact came mostly from the canal. As mentioned in Linsley and Franzini (1979), groundwater is usually cleared of sediments if flowing through fine-grained materials within a distance of $30 \mathrm{~m}$.

There are many analytical and semi-analytical solutions developed for describing groundwater flow in aquifers with a pumping well located near a stream (e.g., Hunt, 1999; Zlotnik and Huang, 1999; Bulter et al., 2001, 2007; Fox et al., 2002; Chen and Yin, 2004; Sun and Zhan, 2007; Yeh et al., 2008; Zlotnik and Tartakovsky, 2008). These studies are however based on the problems with a vertical pumping well which may produce a large drawdown cone near the well.

Since 1930s, the horizontal well was used for avoiding some adverse effects involved in the vertical well. In reality, the horizontal well was not widely adopted due to lack of techniques in drilling the wellbore. Recently, installing horizontal wells has significantly advanced. The installation of vertical wells may not be feasible for the locations where the ground surfaces are covered with obstructions such as buildings and roads. Those problems can be overcome if adopting the horizontal well. In addition, horizontal wells have better contact within the stratum and can be installed in aquifers of small thickness if a long screen length is required. Several previous studies associated with the use of horizontal wells are proposed in the fields of water resources and environmental engineering (e.g., Zhan, 1999; Park and Zhan, 2002, 2003; Zhan and Park, 2003; Kompani-Zare et al., 2005). Zhan et al. (2001) provided an analytical solution to describe flow toward a horizontal well in an anisotropic confined aquifer. Zhan and Zlotnik (2002) extended point source solution to the cases of slanted wells based on the principle of superposition. Joshi (2003) indicated that the use of horizontal wells reduces $50 \%$ operating costs in comparison with

Published by Copernicus Publications on behalf of the European Geosciences Union. 


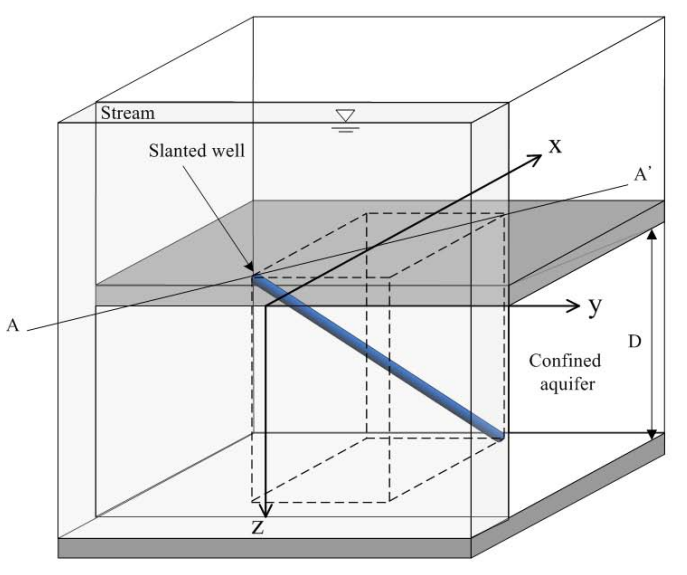

(a)

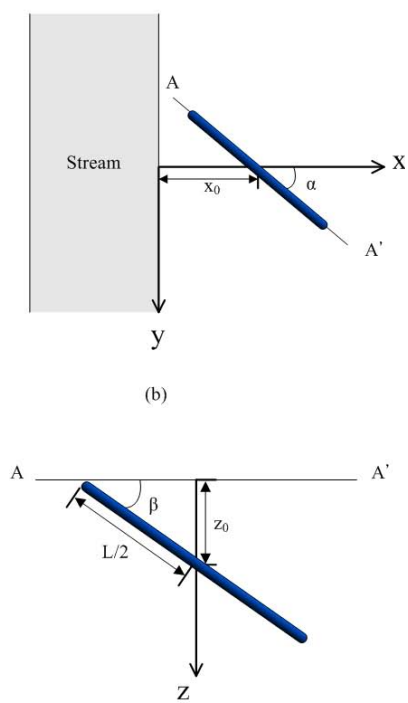

(c)

Fig. 1. Schematic diagram. (a) 3-D view (b) top view (c) view of vertical plane including cross section A-A'

that of vertical wells. Sun and Zhan (2006) mentioned the use of a horizontal well in an aquitard-aquifer system beneath a water reservoir for water supply. Their results illustrate that the leakage induced from the pumping of the horizontal well is dependent on the well location and length as well as the thickness, storage and vertical hydraulic conductivity of the aquitard.

Some articles also addressed the problems of pumping horizontal wells near the stream with considering the stream as the first-type (constant head) or second-type (constant flux) boundary (e.g., Kawecki, 2000; Zhan and Cao, 2000; Langseth et al., 2004). Fox (2007) used a semi-analytical approach and showed how the length of the unsaturated zone under a stream increases in time as a result of pumping. Wang and Zhang (2007) used a numerical simulation for describing the behavior of the infiltration from the stream during pumping of horizontal seepage wells. They indicated that if a streambed has a lower conductivity than the aquifer (e.g., through streambed clogging), a disconnection between surface water and groundwater is likely to occur. Under these circumstances, the use of first-type or second-type boundary condition for the stream is not appropriate.

This paper is first to develop the solutions for describing the behaviors of groundwater flow due to a point source pumping in an anisotropic confined aquifer near a stream. Consider that the water flowing to the well is significantly less than that in the stream; therefore, the effect of depletion on the stream is negligible. Otherwise, some negative impacts due to depletion of the stream had been discussed in Konikow and Kendy (2005). The solutions in terms of head distributions for horizontal and slanted wells are then obtained by integrating the point source solution along the well axis. With the aid of the newly developed solutions, the best orientation and inclination of the well axis can be determined for flow reaching the quasi-steady state. The effects of the aquifer anisotropy, well length, and distance from the well to the stream on the SDR obtained from the horizontal well are also investigated.

\section{Methods}

\subsection{Conceptual model}

Figure 1 shows a three-dimensional (3-D) conceptual model for a confined aquifer with a slanted well near a stream. Assume that the aquifer is homogeneous and anisotropic. The origin of the coordinate system is located at the top of the upper boundary of the aquifer and the intersection between the aquifer and stream. The top of the stream is considered as the reference datum. The aquifer is of a thickness D and semiinfinite extent in $\mathrm{x}$-direction and infinite extent in y-direction. The length of the well is $\mathrm{L}$ and the distance measured from the upper boundary of the aquifer to the middle of the well is $z_{0}$ as shown in Fig. 1c. The distance between the stream and the middle of the well is defined as $x_{0}$ shown in Fig. 1b. The angles $\alpha$ shown in Fig. $1 \mathrm{~b}$ and $\beta$ shown in Fig. 1c represent the orientation and inclination of the well, respectively. Consider two special cases that the slanted well becomes a horizontal well when $\beta=0$ and is perpendicular to the stream when $\alpha=0$ shown in Fig. $2 \mathrm{a}$ and parallel to the stream when $\alpha=90^{\circ}$ shown in Fig. 2 b.

Three assumptions for the development of the mathematical model are made as follows: 1 . The stream stage is not influenced by pumping; 2 . The aquifer is assumed to maintain confined condition; 3 . The hydraulic conductivities of 
(a)
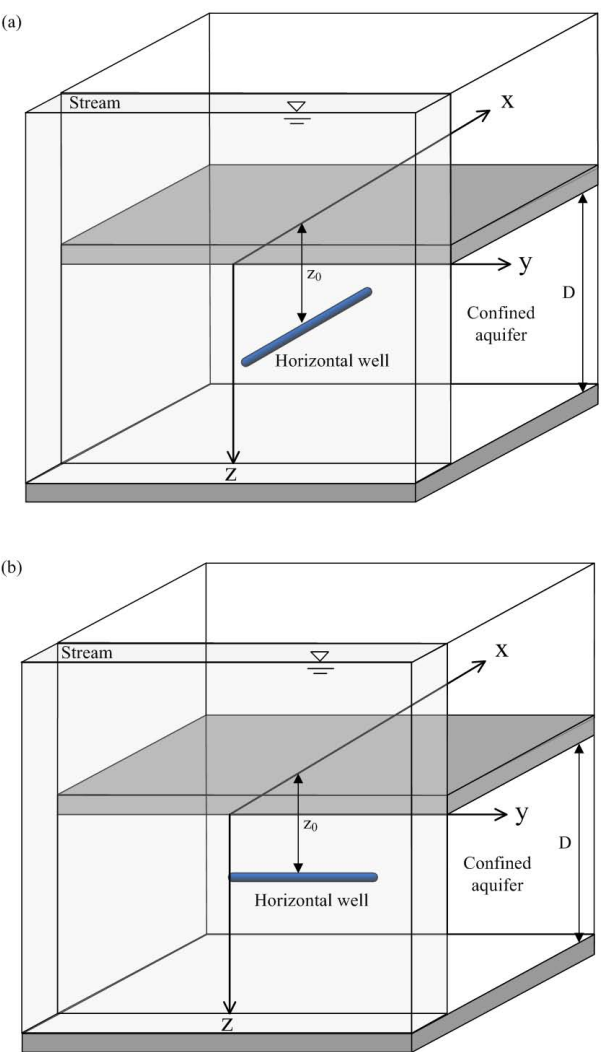
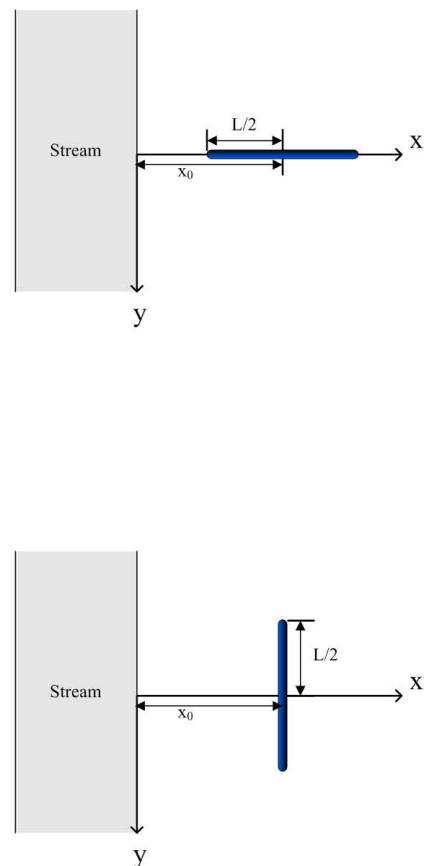

Fig. 2. Schematic diagram of horizontal well for $\beta=0$ and $\alpha=$ (a) 0 (b) $90^{\circ}$

the aquifer and streambed are the same. The first two assumptions imply that the proposed model is applicable if no unsaturated zone (no seepage face) occurs and the pumped water is significantly less than the stream water.

The governing equation for describing 3-D transient head distribution $h(x, y, z, t)$ in a confined aquifer with a point source can be expressed as

$K_{x} \frac{\partial^{2} h}{\partial x^{2}}+K_{y} \frac{\partial^{2} h}{\partial y^{2}}+K_{z} \frac{\partial^{2} h}{\partial z^{2}}=S_{s} \frac{\partial h}{\partial t}$

$+Q \delta\left(x-x_{0}^{\prime}\right) \delta\left(x-y_{0}^{\prime}\right) \delta\left(x-z_{0}^{\prime}\right)$,

where $K_{x}, K_{y}, K_{z}$ are hydraulic conductivities in the x-, y, z-directions, respectively; $S_{s}$ is the specific storage; $\mathrm{Q}$ is the pumping rate for the point source; $\delta()$ is the Dirac delta function and $\left(x_{0}^{\prime}, y_{0}^{\prime}, z_{0}^{\prime}\right)$ is the location of the point source.

The boundary condition along the stream is expressed as

$h(x=0, y, z, t)=0$.

and the boundary conditions at the top and bottom of the confined aquifer are, respectively,

$\frac{\partial h}{\partial z}(x, y, z=0, t)=0$

$\frac{\partial h}{\partial z}(x, y, z=D, t)=0$.
The remote boundary conditions in $\mathrm{x}$ and $\mathrm{y}$ directions are formulated as

$\lim _{x \rightarrow \infty} h=0$

$\lim _{y \rightarrow \pm \infty} h=0$.

The initial condition is

$h(x, y, z, t=0)=0$.

Dimensionless variables are introduced as:

$x_{D}=\frac{x}{D}, \quad y_{D}=\frac{y}{D}, \quad z_{D}=\frac{z}{D}$,

$x_{0 D}=\frac{x_{0}}{D}, \quad y_{0 D}=\frac{y_{0}}{D}, \quad z_{0 D}=\frac{z_{0}}{D}$,

$t_{D}=\frac{K_{x}}{D^{2} S_{s}} t, \quad L_{D}=\frac{L}{D}, \quad h_{D}=\frac{\pi^{2} K_{x} D}{Q} h$.

According to Eq. (8), the governing equation can be rewritten as

$$
\begin{aligned}
& \frac{\partial^{2} h_{D}}{\partial x_{D}^{2}}+\kappa_{y} \frac{\partial^{2} h_{D}}{\partial y_{D}^{2}}+\kappa_{z} \frac{\partial^{2} h_{D}}{\partial z_{D}^{2}} \\
& =\frac{\partial h_{D}}{\partial t_{D}}+\pi^{2} \delta\left(x-x_{0}^{\prime}\right) \delta\left(x-y_{0}^{\prime}\right) \delta\left(x-z_{0}^{\prime}\right)
\end{aligned}
$$

where $\kappa_{y}=K_{y} / K_{x}$ and $\kappa_{z}=K_{z} / K_{x}$. 
The boundary and initial conditions (2-7) become

$$
\begin{aligned}
& h_{D}\left(x_{D}=0, y_{D}, z_{D}, t_{D}\right)=0 \\
& \frac{\partial h_{D}}{\partial z_{D}}\left(x_{D}, y_{D}, z_{D}=0, t_{D}\right)=0 \\
& \frac{\partial h_{D}}{\partial z_{D}}\left(x_{D}, y_{D}, z_{D}=1, t_{D}\right)=0 \\
& \lim _{x_{D} \rightarrow \infty} h_{D}=0 \\
& \lim _{y_{D} \rightarrow \pm \infty} h_{D}=0 \\
& h_{D}\left(x_{D}, y_{D}, z_{D}, t_{D}=0\right)=0 .
\end{aligned}
$$

\subsection{Transient head solutions}

Applying Fourier transforms to Eqs. (9-15) and then inverting the solution in Fourier domain results in

$h_{D}\left(x_{D}, y_{D}, z_{D}, t_{D}\right)=2 \int_{0}^{\infty} \int_{0}^{\infty}\left(\Phi_{0}+\sum_{n=1}^{\infty} \Phi_{n}\right) d \xi d \omega$

with

$\Phi_{0}=\frac{\sin \left(\omega x_{0}^{\prime}\right) \sin \left(\omega x_{D}\right) \cos \left[\left(y_{0}^{\prime}-y_{D}\right) \xi\right]\left(e^{-\left(\omega^{2}+\kappa_{y} \xi^{2}\right) t_{D}}-1\right)}{\omega^{2}+\kappa_{y} \xi^{2}}$

and

$\Phi_{n}$

$=\frac{2 \sin \left(\omega x_{0}^{\prime}\right) \sin \left(\omega x_{D}\right) \cos \left(n \pi z_{0}^{\prime}\right) \cos \left(n \pi z_{D}\right) \cos \left[\left(y_{0}^{\prime}-y_{D}\right) \xi\right]\left(e^{-\left(\omega^{2}+\kappa_{y} \xi^{2}+\kappa_{z} n^{2} \pi^{2}\right) t_{D}}-1\right)}{\omega^{2}+\kappa_{y} \xi^{2}+\kappa_{z} n^{2} \pi^{2}}$

where $\omega, \xi$ and $n$ are the variables of Fourier sine, Fourier and finite Fourier cosine transforms, respectively. Note that Eq. (18) contains $z_{0}^{\prime}$ which reflects the influence of the depth of the point source on the head distribution in Eq. (16). For detailed derivation of Eq. (16), readers are referred to Appendix A.

Based on the principle of superposition, integrating Eq. (16) along slanted well axis leads to

$h_{D s}\left(x_{D}, y_{D}, z_{D}, t_{D}\right)=\frac{1}{L_{D}} \int_{-\frac{L_{D}}{2}}^{\frac{L_{D}}{2}} h_{D}\left(x_{D}, y_{D}, z_{D}, t_{D}\right) d l$

with

$x_{0}^{\prime}=l \cos (\alpha) \cos (\beta)+x_{0 D}$,

$y_{0}^{\prime}=l \sin (\alpha) \cos (\beta)$

and

$z_{0}^{\prime}=l \sin (\beta)+z_{0 D}$

where $l$ is a dummy variable for integration along the slanted well axis.

\subsection{Stream depletion rate for pumping in confined aquifers}

The SDR herein is defined as the ratio of the quantity of water obtained from the stream to the total amount of water from the well per unit time. The stream is assumed to be hydraulically connected with the adjacent aquifer. Therefore, the SDR determined from Darcy's law for the slanted well can be expressed as

$\frac{q\left(t_{D}\right)_{s}}{Q}=\frac{1}{\pi^{2}} \int_{-\infty}^{\infty} \int_{0}^{1} \frac{\partial h_{D s}}{\partial x_{D}} d z_{D} d y_{D} \quad$ at $\quad x_{D}=0$

Substituting Eq. (19) into Eq. (23) results in

$\frac{q\left(t_{D}\right)_{s}}{Q}$

$=\frac{2}{\pi^{2} L_{D}} \int_{-\infty}^{\infty} \int_{-\frac{L_{D}}{2}}^{\frac{L_{D}}{2}} \int_{0}^{\infty} \int_{0}^{\infty} \frac{\omega \sin \left(\omega x_{0}^{\prime}\right) \cos \left[\xi\left(y_{0}^{\prime}-y_{D}\right)\right]\left(e^{-\left(\omega^{2}+\kappa_{y} \xi^{2}\right) t_{D}}-1\right)}{\omega^{2}+\kappa_{y} \xi^{2}} d \omega d \xi d l d y_{D}$

where $x_{0}^{\prime}$ and $y_{0}^{\prime}$ are defined in Eqs. (20) and (21), respectively. Equation (23) developed based on Eq. (16) implicitly includes the term $\Phi_{n}$ in Eq. (16). The result of integration to $z_{D}$ for $\Phi_{n}$ in Eq. (23) is zero, indicating that the SDR is independent of the depth of the well.

\subsection{Quasi-steady state solution}

The drawdown due to pumping increases with time and approaches steady state after a certain period of time. This is because the exponential terms in Eqs. (17) and (18) become small, say, less than $10^{-4}$, when the time is large. A quasisteady solution can then be obtained by applying the residue theorem when neglecting the exponential terms in Eqs. (17) and (18). The development of quasi-steady state solution form Eq. (16) is shown in Appendix A and the result is

$h_{q}\left(x_{D}, y_{D}, z_{D}\right)=-2 \int_{0}^{\infty}\left(\frac{1}{2} C_{0}+\sum_{n=1}^{\infty} C_{n}\right) d \omega$

where

$C_{0}=-\frac{\sin \left(\omega x_{0}^{\prime}\right) \sin \left(\omega x_{D}\right) e^{-\left|y_{0}^{\prime}-y_{D}\right| \sqrt{\frac{\omega^{2}}{\kappa_{y}}}}}{\sqrt{\frac{\omega^{2}}{\kappa_{y}}}}$

and

$$
=\frac{\sin \left(\omega x_{0}^{\prime}\right) \sin \left(\omega x_{D}\right) \cos \left(n \pi z_{0}^{\prime}\right) \cos \left(n \pi z_{D}\right) e^{-\left|y_{0}^{\prime}-y_{D}\right| \sqrt{\frac{\omega^{2}}{\kappa_{y}}+\frac{\kappa_{z} n^{2} \pi^{2}}{\kappa_{y}}}}}{\sqrt{\frac{\omega^{2}}{\kappa_{y}}+\frac{\kappa_{z} n^{2} \pi^{2}}{\kappa_{y}}}}
$$

Based on the principle of superposition, integrating Eq. (25) along the horizontal and vertical well axes yields the 


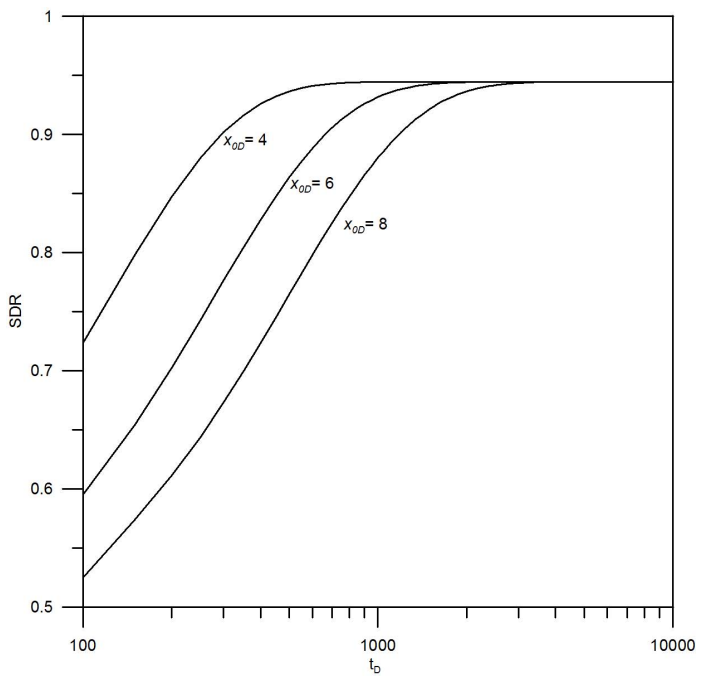

Fig. 3. The SDR from the horizontal well parallel to the stream versus dimensionless time for $\kappa_{y}=1, \kappa_{z}=1, L_{D}=1$, and various distance between the well and stream.

hydraulic head distributions in quasi-steady state, respectively, as

$H_{h}\left(x_{D}, y_{D}, z_{D}\right)=\frac{1}{L_{D}} \int_{-\frac{L_{D}}{2}}^{\frac{L_{D}}{2}} h_{q}\left(x_{D}, y_{D}, z_{D}\right) d y_{0}^{\prime}$

and

$H_{v}\left(x_{D}, y_{D}, z_{D}\right)=\frac{1}{L_{D}} \int_{-\frac{L_{D}}{2}}^{\frac{L_{D}}{2}} h_{q}\left(x_{D}, y_{D}, z_{D}\right) d z_{0}^{\prime}$.

For the evaluation of Eq. (28), we assume $x_{0}^{\prime}=x_{0 D}$ in Eq. (26) as well as $x_{0}^{\prime}=x_{0 D}$ and $z_{0}^{\prime}=z_{0 D}$ in Eq. (27). Similarly, we assume $x_{0}^{\prime}=x_{0 D}$ and $y_{0}^{\prime}=y_{0 D}$ in both Eqs. (26) and (27) for the evaluation of Eq. (29).

\section{Results and Discussion}

\subsection{Effects of well length and distance on SDR}

Figure 3 shows the SDR from the horizontal well parallel to the stream versus $t_{D}$ for $\kappa_{y}=1, \kappa_{z}=1, L_{D}=1(L=10$ $\mathrm{m})$, and various dimensionless distance $x_{0 D}$. The curve of $x_{0 D}=8\left(x_{0}=80 \mathrm{~m}\right)$ shows that the SDR approaches constants when $t_{D}$ exceeds $1200(t=11.5 \mathrm{~min})$, indicating that the present solution reaches quasi-steady state. The figure indicates that the well with a smaller $x_{0 D}$ has a higher SDR and reaches the quasi-steady state more quickly. This is because the smaller distance between the stream and well, the less time required for the stream water reaching the well. Therefore, the distance $x_{0 D}$ is an important factor in affecting the SDR.

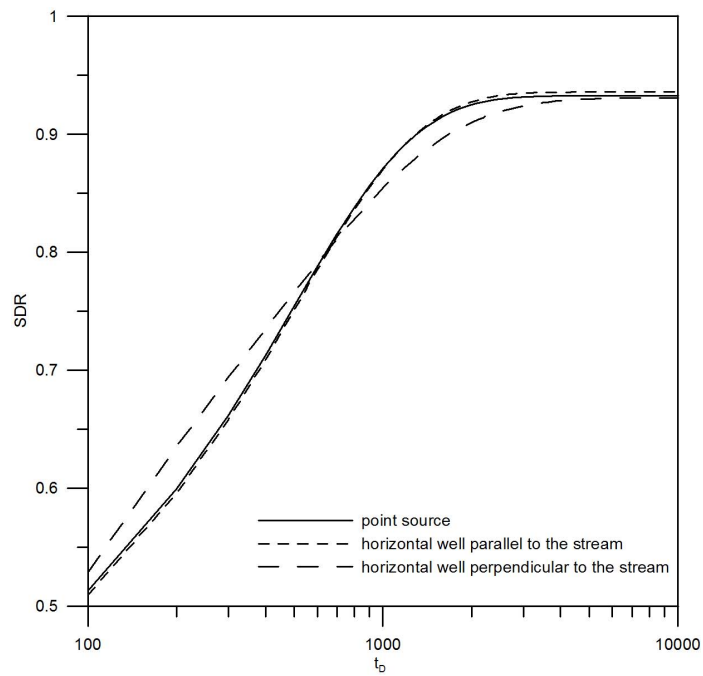

Fig. 4. Comparison of the SDR obtained from the point source and the horizontal well parallel and perpendicular to the stream for $\kappa_{y}=1, \kappa_{z}=1, x_{0 D}=8$ and $L_{D}=10$.

Figure 4 shows the curves of the SDR versus the dimensionless time $t_{D}$ for the point source as well as the horizontal well parallel and perpendicular to the stream for $\kappa_{y}=1$, $\kappa_{z}=1, x_{0 D}=8$ and $L_{D}=10(L=100 \mathrm{~m})$. The dimensionless well length $L_{D}$ may range from 1 to 10 when the thickness of the aquifer is $10 \mathrm{~m}$. When $L_{D}=10$, the difference in predicted SDR between the point source solution and the solution of the horizontal well parallel to the stream is very small, indicating that the effect of well length on the SDR is negligible. Such a result can be attributed to the fact that the stream is considered as infinitely extended and the horizontal well can therefore be considered to be a point source even if the well length is long. The SDR predicted from the point source solution is smaller than that predicted from the solution of the horizontal well perpendicular to the stream when $t_{D}$ is in the range of 100 600 ( $t: 0.96 \sim 5.76 \mathrm{~min})$. This is because the horizontal well extends the half well length toward the stream and thus is relatively closer to the stream than the location of the point source. However, the SDR from the solution of the horizontal well perpendicular to the stream is smaller when $t_{D}>600(t>5.76 \mathrm{~min})$ and reaches quasi-steady state more slowly than that from the point source. This is because the other half of the horizontal well extending toward inland has a longer distance measured from the stream. Therefore, the effect of the well length on the SDR is small.

\subsection{Best orientation $\alpha$ and inclination $\beta$ for less time re- quired for reaching quasi-steady state}

Figure 5 shows the contour for the dimensionless time required for reaching quasi-steady state for $\kappa_{y}=1, \kappa_{z}=$ $1, x_{0 D}=4\left(x_{0}=40 \mathrm{~m}\right), L_{D}=1$, and various orientation $\alpha$ and inclination $\beta$. This figure shows that the smallest 


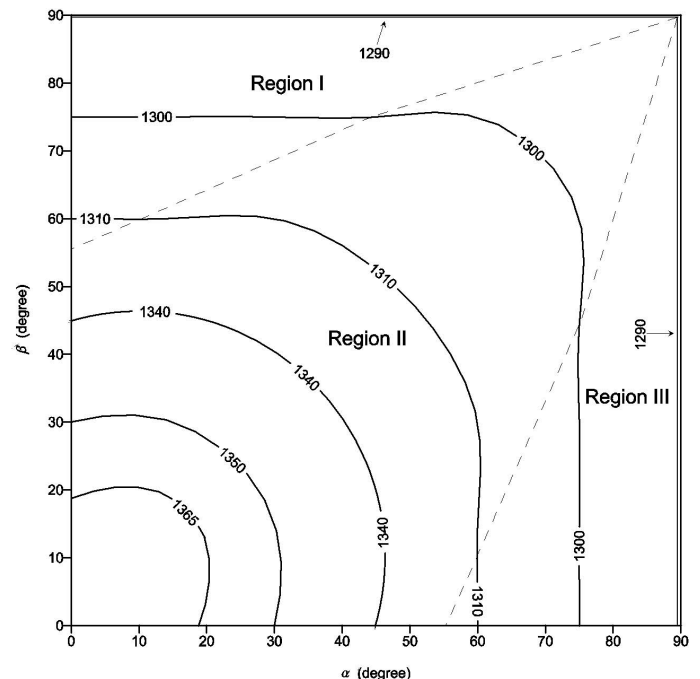

Fig. 5. Contours for the time for reaching quasi-steady state SDR for $\kappa_{y}=1, \kappa_{z}=1, x_{0 D}=4, L_{D}=1$, and various orientation $\alpha$ and inclination $\beta$.

dimensionless time is $1290(t=12.38 \mathrm{~min})$ for $\alpha=90^{\circ}$ or $\beta=90^{\circ}$, which indicates the smallest time for the groundwater flow system reaching quasi-steady state occurs in the case of the vertical well or horizontal well parallel to the stream. The dashed lines divide this figure into three regions. The contour lines in the region I for $\beta$ close to $90^{\circ}$ are almost horizontal, indicating that the effect of $\alpha$ on the time of reaching quasi-steady state is negligible. In contrast, the contour lines in the region III for $\alpha$ near $90^{\circ}$ are nearly vertical, indicating $\beta$ has little influence on the time for reaching quasi-steady state. In the region II, both $\alpha$ and $\beta$, however, have obvious effect on the time for reaching quasi-steady state. Therefore, the smallest time for reaching quasi-steady state occurs in the case of $\alpha=90^{\circ}$ or $\beta=90^{\circ}$.

\subsection{Effect of anisotropic aquifer on the time required for reaching quasi-steady state}

The aquifer near the stream is very likely anisotropic due to the sediment depositional process over a long period of time. The curves of the time for reaching quasi-steady state for $x_{0 D}=4, L_{D}=1$, and various values of $K_{x}$ and $K_{y}$ are shown in Fig. 6. All of the curves are vertical indicating that $K_{y}$ has no influence on the time for reaching quasi-steady state. Obviously, the $K_{x}$ is the key factor in influencing the time for reaching quasi-steady state and the SDR reaches quasisteady state more quickly when $K_{x}$ is large.

\subsection{Drawdown of horizontal and vertical wells}

Figure 7 shows the curves of the quasi-steady head distributions versus dimensionless distance $x_{D}$ for $\kappa_{y}=1, \kappa_{z}=1$, $x_{0 D}=4, L_{D}=1$, and various dimensionless horizontal well

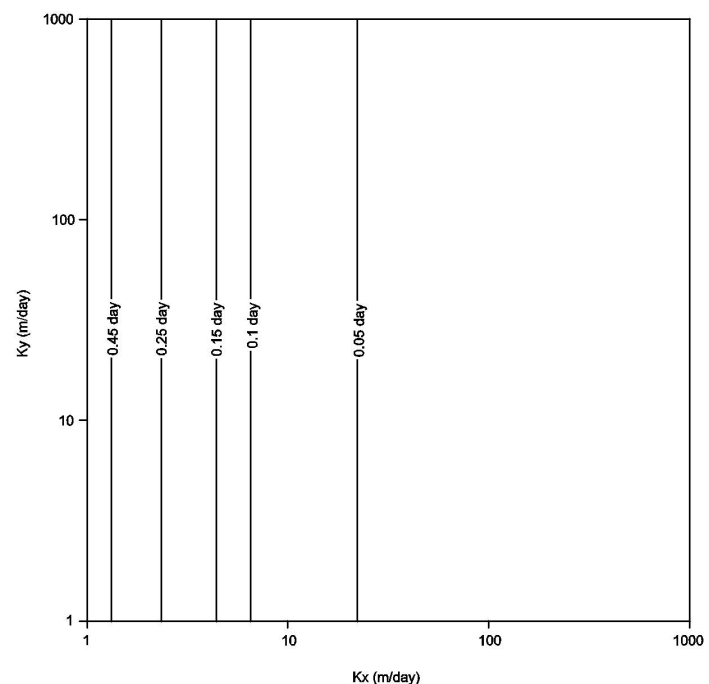

Fig. 6. Contours for the time for reaching quasi-steady state SDR for $x_{0 D}=4, L_{D}=1$, and various hydraulic conductivities $K_{x}$ and $K_{y}$.

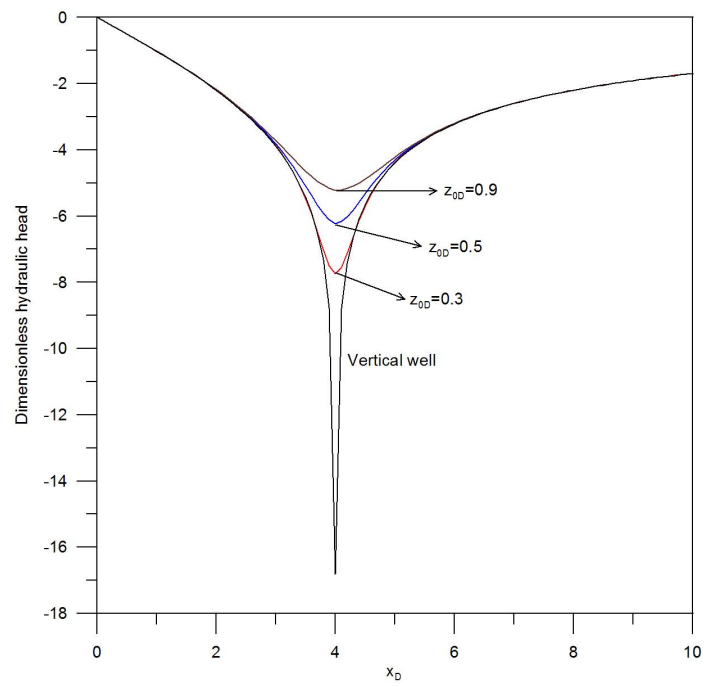

Fig. 7. The spatial head distribution for $\kappa_{y}=1, \kappa_{z}=1, x_{0 D}=4$, $L_{D}=1$, and various depth of the horizontal well parallel to the stream.

depth $z_{0 D}$. This figure shows that the drawdown produced by the horizontal well is smaller than that by the vertical well of full penetration when $z_{0 D}$ is larger than $0.3\left(z_{0}=3 \mathrm{~m}\right)$. In addition, the figure also shows that the drawdown will be smaller if the horizontal well is deeper. On the other hand, Fig. 8 demonstrates the dimensionless head distribution versus $x_{D}$ for $\kappa_{y}=1, \kappa_{z}=1, x_{0 D}=4, z_{0 D}=0.2\left(z_{0}=2 \mathrm{~m}\right)$, and various $L_{D}$. The drawdown produced by the horizontal well is significantly smaller than that by the vertical well of full penetration when $L_{D}>1.4(L>14 \mathrm{~m})$, indicating that a larger $L_{D}$ results in a smaller drawdown. In addition, the 


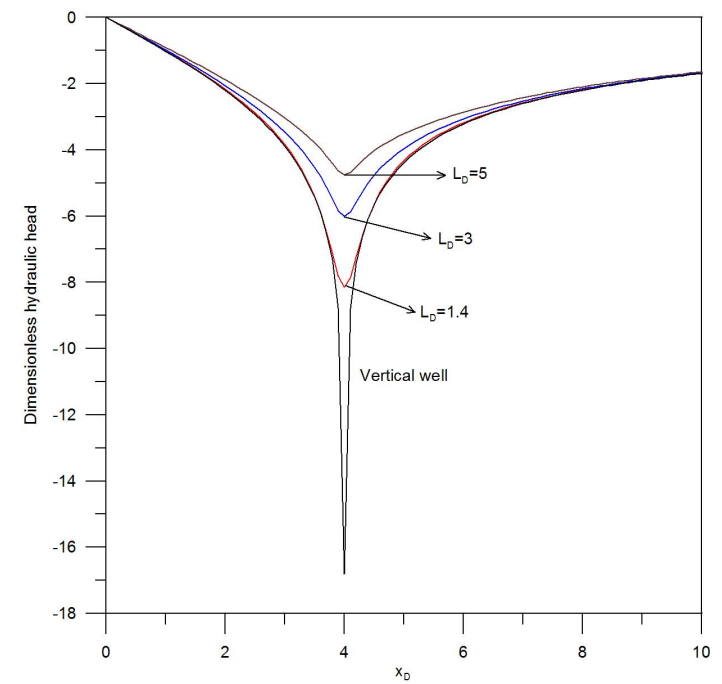

Fig. 8. The spatial head distribution for $\kappa_{y}=1, \kappa_{z}=1, x_{0 D}=$ $4, z_{0 D}=0.2$, and various length of the horizontal well parallel to the stream.

horizontal well with a large $L_{D}$ produces shallow drawdown cones of wide extent as shown in Fig. 8, while the deep horizontal well produces tight drawdown cones as shown in Fig. 7. This indicates that the use of a long well length in producing small drawdown is more effective than that of a deep horizontal well.

\section{Concluding remarks}

A 3-D point source solution for describing the head distribution of the confined aquifer near a stream is developed by applying Fourier transforms. The aquifer is assumed to be homogenous and the nearby stream is considered as a constanthead boundary. Integrating the point source solution along the well axis yields the analytical solutions for the horizontal and slanted wells. In addition, the quasi-steady solutions can be obtained by neglecting the exponential terms in the solutions. The effects of the horizontal well length and distance between the well and stream on the SDR are investigated. The best direction of well axis can be determined from the smallest time required for reaching the quasi-steady groundwater flow system. In addition, the influences of anisotropy on the time for reaching quasi-steady state are also addressed. The following conclusions can be drawn from this study:

1. The SDR is almost independent on the length of the horizontal well. The SDR reaches quasi-steady state more quickly if the well is closer to the stream.

2. To avoid producing large drawdown and have less time for reaching quasi-steady SDR, the horizontal well would be better to install parallel to the stream.
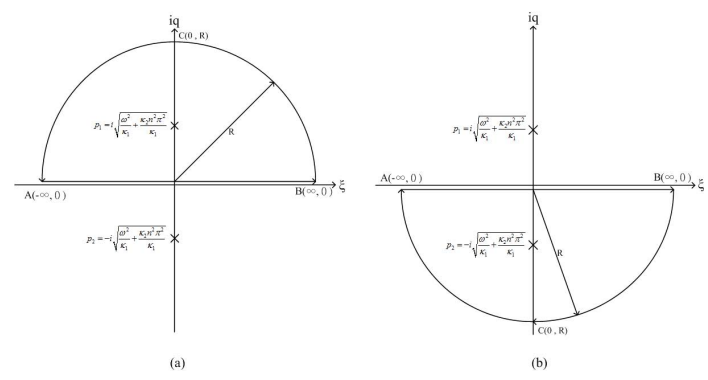

Fig. 9. Contour of integration.

3. The $K_{x}$ plays the key role in effecting the time for reaching quasi-steady SDR while $K_{y}$ has no influence on the time for reaching quasi-steady SDR.

4. The use of a long horizontal well in producing small drawdown is better than that of a deep one under a constant pumping rate.

\section{Appendix A}

\section{Mathematical background}

Applying finite Fourier cosine, Fourier sine and Fourier transforms to the variables $z_{D}, x_{D}$ and $y_{D}$ in Eqs. (9$15)$, respectively, leads to a first-order ordinary differential equation and initial condition in term of $t_{D}$. Note that $\lim _{x_{D} \rightarrow \infty} \partial h_{D} / \partial x_{D}=0$ and $\lim _{y_{D} \rightarrow \pm \infty} \partial h_{D} / \partial y_{D}=0$ (remote boundary conditions) are also required in Fourier sine and Fourier transforms, respectively. Solving the ordinary differential equation and then using inverse Fourier transform yield Eq. (16).

The quasi-steady state solution by neglecting the exponential terms in Eqs. (17) and (18) can be expressed in terms of imaginary unit $i$ as

$h_{q}\left(x_{D}, y_{D}, z_{D}\right)=-2 \int_{0}^{\infty}\left(\frac{1}{2} \Omega_{0}+\sum_{n=1}^{\infty} \Omega_{n}\right) d \omega$

in which

$\Omega_{0}=\operatorname{Re}\left[\int_{-\infty}^{\infty} \frac{\sin \left(\omega x_{0}^{\prime}\right) \sin \left(\omega x_{D}\right) e^{\left(y_{0}^{\prime}-y_{D}\right) \xi i}}{\omega^{2}+\kappa_{y} \xi^{2}} d \xi\right]$

and

$\Omega_{n}=R e\left[\int_{-\infty}^{\infty} \frac{\sin \left(\omega x_{0}^{\prime}\right) \sin \left(\omega x_{D}\right) \cos \left(n \pi z_{0}^{\prime}\right) \cos \left(n \pi z_{D}\right) e^{\left(y_{0}^{\prime}-y_{D}\right) \xi i}}{\omega^{2}+\kappa_{y} \xi^{2}+\kappa_{z} n^{2} \pi^{2}} d \xi\right]$

where Re represents the real part of the left-hand side terms in Eqs. (A2) and (A3). 
A close path of integration shown in Fig. 9 with a straight line parallel to the real axis and semicircle BCA is constructed for the integrations of Eq. (A3). According to Jordan's Lemma (Folland, 2002), the integration over the semicircular path BCA in Fig. 9a tends to zero when $y_{0}^{\prime}-y_{D}>0$ and $R \rightarrow \infty$. In contrast, the result of integration over the path BCA in Fig. $9 \mathrm{~b}$ always tends to zero when $y_{0}^{\prime}-y_{D}<0$ and $R \rightarrow \infty$.

Based on the residue theorem, the result of integration for Eq. (A3) can be expressed as

$\Omega_{n}=\left.2 \pi i \operatorname{Res}\right|_{\xi=p_{1}}$ for $y_{0}^{\prime}-y_{D}>0$

and

$\Omega_{n}=\left.2 \pi i \operatorname{Res}\right|_{\xi=p_{2}}$ for $y_{0}^{\prime}-y_{D}<0$

where $\quad p_{1}$ and $\quad p_{2}$, singularities, represent $i \sqrt{\left(\omega^{2}+\kappa_{z} n^{2} \pi^{2}\right) / \kappa_{y}}$ and $-i \sqrt{\left(\omega^{2}+\kappa_{z} n^{2} \pi^{2}\right) / \kappa_{y}}$ in complex plane, respectively. The residues of the singularities can be estimated by following formulas (Kreyszig, 1999)

$$
\left.\operatorname{Res}\right|_{\xi=p_{1}}=\lim _{\xi=p_{1}} \Omega_{n} \cdot\left(\xi-p_{1}\right) \text { for } y_{0}^{\prime}-y_{D}>0
$$

and

$$
\left.\operatorname{Res}\right|_{\xi=p_{2}}=\lim _{\xi=p_{2}} \Omega_{n} \cdot\left(\xi-p_{2}\right) \text { for } y_{0}^{\prime}-y_{D}<0
$$

Substituting Eqs. (A6) and (A7) into Eqs. (A4) and (A5), respectively and taking the real part of these two results yield

$$
\begin{aligned}
& \Omega_{n}= \\
& \frac{\sin \left(\omega x_{0}^{\prime}\right) \sin \left(\omega x_{D}\right) \cos \left(n \pi z_{0}^{\prime}\right) \cos \left(n \pi z_{D}\right) e^{-\left(y_{0}^{\prime}-y_{D}\right) \sqrt{\frac{\omega^{2}}{\kappa_{y}}+\frac{\kappa_{z} n^{2} \pi^{2}}{\kappa_{y}}}}}{\sqrt{\frac{\omega^{2}}{\kappa_{y}}+\frac{\kappa_{z} n^{2} \pi^{2}}{\kappa_{y}}}} \text { for } y_{0}^{\prime}-y_{D}>0
\end{aligned}
$$

and

$$
\begin{aligned}
& \Omega_{n}= \\
& \frac{\sin \left(\omega x_{0}^{\prime}\right) \sin \left(\omega x_{D}\right) \cos \left(n \pi z_{0}^{\prime}\right) \cos \left(n \pi z_{D}\right) e^{\left(y_{0}^{\prime}-y_{D}\right) \sqrt{\frac{\omega^{2}}{\kappa_{y}}+\frac{\kappa_{z} n^{2} \pi^{2}}{\kappa_{y}}}}}{\sqrt{\frac{\omega^{2}}{\kappa_{y}}+\frac{\kappa_{z} n^{2} \pi^{2}}{\kappa_{y}}}} \text { for } y_{0}^{\prime}-y_{D}<0
\end{aligned}
$$

The exponential term in Eq. (A9) is equal to that in Eq. (A8) when the term $y_{0}^{\prime}-y_{D}$ in Eq. (A9) is less than zero. Therefore, the integration of Eq. (A3) results in Eq. (27). Otherwise, substituting $n=0$ into Eq. (27) leads to Eq. (26) which is the result of integration of Eq. (A2).

Acknowledgements. This study was partly supported by the Taiwan National Science Council under the grant NSC 97-2313-B-005042-MY3.

\section{Edited by: H. Cloke}

\section{References}

Butler, J. J., Zlotnik, B. A. and Tsou, M. S.: Drawdown and stream depletion produced by pumping in the vicinity of a partially penetrating stream, Ground Water, 39(5), 651-659, 2001.

Brunner, P., Cook, P. G., and Simmons, C. T.: Hydrogeologic controls on disconnection between surface water and groundwater, Water Resour. Res., 45(1), W01422, doi:10.1029/2008WR006953, 2009.

Butler, J. J., Zhan, X., and Zlotnik, V. A.: Pumping-Induced Drawdown and Stream Depletion in a Leaky Aquifer System, Ground Water, 45(2), 178-186, doi:10.1111/j.1745-6584.2006.00272.x, 2007.

Chen, X. and Yin, Y.: Semianalytical solutions for stream depletion in partially penetrating streams, Ground Water, 42(1), 9296, 2004.

Folland, G. B.: Advanced calculus, Prentice Hall, New Jersey, 2002.

Fox, G. A., DuChateau, P., and Durnford, D. S.: Analytical Model for aquifer response incorporating distributed stream leakage, Ground Water, 40(4), 378-384, 2002.

Fox, G. A. and Gordji, L.: Consideration for unsaturated flow beneath a streambed during alluvial well depletion, J. Hydrol. Eng., 12(2), 139-145, doi:10.1061/(ASCE)10840699(2007)12:2(139), 2007.

Hunt, B.: Unsteady stream depletion from ground water pumping, Ground Water, 37(1), 98-102, 1999.

Joshi, S. D.: Cost/Benefits of horizontal wells, Society of Petroleum Engineers, 19-24, 2003.

Kawecki, M. W.: Transient flow to a horizontal water well, Ground Water, 38(6), 842-850, 2000.

Kompani-Zare, M., Zhan, H., and Samani, N.: Analytical study of capture zone of a horizontal well in a confined aquifer, J. Hydrol., 307(1-4), 48-59, doi:10.1016/j.hydrol.2004.09.021, 2005.

Kreyszig, E.: Advanced engineering mathematics, John Wiley \& Sons Inc., New York, 1999.

Konikow, L. F. and Kendy, E.: Groundwater depletion: A global problem, Hydrogeol. J., 13(1), 317-320, doi:10.1007/s10040004-0411-8, 2005.

Langseth, D. E., Smyth, A. H., and May, J.: A method for evaluating horizontal well pumping tests, Ground water, 42(5), 689-699, 2004.

Linsley, R. K. and Franzini, J. B.: Water resources engineering, McGraw-Hill Book Company, New York, 1979.

Park, E. and Zhan, H.,: Hydraulics of a finite-diameter horizontal well with wellbore storage and skin effect in leady aquifers, Adv. Water Resour., 25, 389-400, 2002.

Park, E. and Zhan, H.: Hydraulics of horizontal well in fractured shallow aquifer systems, J. Hydrol., 281, 147-158, 2003.

Sun, D. and Zhan, H.: Flow to a horizontal well in an aquitard-aquifer system, J. Hydrol., 321(1-4), 364-376, doi:10.1016/j.jhydrol.2005.08.008, 2006.

Sun, D. and Zhan, H.: Pumping induced depletion from two streams, Adv. Water Resour., 30(4), 1016-1026, doi:10.1016/j.advwatres.2006.09.001, 2007.

Wang, W. and Zhang, G.: Numerical simulation of groundwater flowing to horizontal seepage wells under a river, Hydrogeol. J., 15(6), 1211-1220, doi:10.1007/s10040-007-0171-3, 2007.

Yeh, H. D., Chang, Y. C., and Zlotnik, V. A.: Stream depletion rate and volume from groundwater pumping in 
wedge-shape aquifers, J. Hydrol., 349(3-4), 501-511, doi:10.1016/j.jhydrol.2007.11.025, 2008.

Zhan, H.: Analytical study of capture time to a horizontal well, J. Hydrol., 217(1-2), 46-54, 1999.

Zlotnik, V. A. and Huang, H.: Effect of shallow penetration and streambed sediments on aquifer response to stream stage fluctuations (analytical model), Ground Water, 37(4), 599-605, 1999.

Zhan, H. and Cao, J.: Analytical and semi-analytical solutions of horizontal well capture times under no-flow and constant-head boundaries, Adv. Water Resour., 23(8), 835-848, 2000.
Zhan, H., Wang, L. V., and Park, E.: On the horizontal-well pumping tests in anisotropic confined aquifers, J. Hydrol., 252(1-4), 37-50, 2001.

Zhan, H. and Zlotnik, V. A.: Ground water flow to horizontal and slanted wells in unconfined aquifers, Water Resour. Res., 38, 1108, doi:10.1029/2001WR000401, 2002.

Zhan, H. and Park, E.: Hydraulics of horizontal wells in leaky aquifers, J. Hydrol, 281, 129-146, 2003.

Zlotnik, V. A. and Tartakovsky, D. M.: Stream depletion by groundwater pumping in leaky aquifers, J. Hydrol. Eng., 13(2), 43-50, doi:10.1061/(ASCE)1084-0699(2008)13:2(43), 2008. 